\title{
Combination of Biochanin A and Temozolomide Impairs Tumor Growth by Modulating Cell Metabolism in Glioblastoma Multiforme
}

\author{
VILAS DESAI ${ }^{1}$, ADITI JAIN ${ }^{2}$, HOORA SHAGHAGHI $^{3}$, ROSS SUMMER ${ }^{3}$, JAMES C. K. LAI ${ }^{4}$ and ALOK BHUSHAN ${ }^{1}$ \\ ${ }^{1}$ Department of Pharmaceutical Sciences, Jefferson College of Pharmacy, \\ Thomas Jefferson University, Philadelphia, PA, U.S.A.; \\ ${ }^{2}$ Department of Surgery, Thomas Jefferson University, Philadelphia, PA, U.S.A.; \\ ${ }^{3}$ Center for Translational Medicine and Jane and Leonard Korman Lung Center, \\ Thomas Jefferson University, Philadelphia, PA, U.S.A.; \\ ${ }^{4}$ Department of Biomedical and Pharmaceutical Sciences, College of Pharmacy and ISU Biomedical Research Institute, \\ Division of Health Sciences, Idaho State University, Pocatello, ID, U.S.A.
}

\begin{abstract}
Background/Aim: Several epidemiological studies have reported the chemopreventive potential of biochanin $A$, in cancer development and progression. We investigated the anticancer potential of combination of biochanin $A$ and temozolomide against U-87 MG and T98 G [glioblastoma multiforme (GBM)] cells. Materials and Methods: We evaluated the effect of biochanin A and temozolomide treatment on cell viability, expression of survival proteins, cell cycle, cell metabolism and mitochondrial function. Results: Enhanced inhibitory effects of the combination treatment were observed on cell viability, expression of cell survival proteins EGFR, $p$ ERK, $p-A K T, c-m y c$ and MT-MMP1, and increased expression of the tumor suppressor, p-p53. Combination treatment also induced arrest in the $G_{1}$ phase of the cell cycle. A shift in the metabolic phenotype of cells from glycolytic to oxidative phosphorylation was observed on combination treatment and the permeabilized cells showed a significant impairment in complex IV activity. Conclusion: Biochanin A significantly enhanced the anticancer efficacy of temozolomide in GBM cells.
\end{abstract}

Glioblastoma multiforme (GBM) is the most common, lethal, primary malignant brain tumor, classified as a Grade

This article is freely accessible online.

Correspondence to: Alok Bhushan, Jefferson College of Pharmacy, Thomas Jefferson University, 901 Walnut Street, Suite 915, Philadelphia, PA 19107, U.S.A. Tel: +1 2155033082, e-mail: Alok.Bhushan@jefferson.edu

Key Words: Glioblastoma, biochanin A, temozolomide, isoflavone, metabolism.
IV astrocytoma by the World Health Organization (WHO) (1). GBM is typically characterized by aggressive cell proliferation, invasion of normal brain tissue and increased angiogenesis $(2,3)$. The current therapy of surgical resection followed by radiotherapy coupled with temozolomide has only minimally improved the median survival time of patients from 12 months to $\sim 14.6$ months $(3,4)$. The poor prognosis of GBM calls for more effective, targeted therapies as well as improvements to the existing therapies.

A promising approach to improve the efficacy of existing therapies is to sensitize the GBM cells to radiation and chemotherapy regimens, such as temozolomide. Temozolomide is an alkylating agent that is lipophilic in nature and has reasonable penetration across the blood-brain barrier (4-8). It acts by adding methyl groups to the $\mathrm{O}^{6}$ position of guanine which leads to the insertion of a thymine in place of cytosine, resulting in a mis-matched base pair that can lead to cell death $(7,8)$. The induction of DNA damage by temozolomide is followed by cell cycle arrest in the $\mathrm{G}_{2} / \mathrm{M}$ phase, eventually causing cell senescence, or apoptosis (5, 9). However, the methylated sites can be fixed by the induction of DNA repair systems, such as the mis-match repair (MMR) or the base excision repair (BER) $(6,7)$. The resistance of GBM to temozolomide has been associated with the expression of a dealkylating enzyme, $\mathrm{O}^{6}$ methylguanine methyltransferase (MGMT) that reverses methylation at the $\mathrm{O}^{6}$ position of guanine and neutralizes the cytotoxic effects of temozolomide (6-8). Thus, resistance to temozolomide and subsequent upregulation of pro-survival \& pro-invasive pathways has evoked interest in evaluating combination therapies against GBM (9-12).

Several studies have reported the role of dietary compounds like isoflavones in influencing the development 
and progression of cancer (13-17). Isoflavones possess potent anti-oxidant and anti-inflammatory properties $(14,18$ 20) and are effective adjuvant chemotherapeutic and radiotherapeutic agents for multiple cancers (15, 17). Epidemiological studies indicate reduced rates of cancers in Asian populations that consume a diet rich in soy $(18,20)$. Isoflavones have been shown to inhibit APE1/REF-1 repair and redox activity that can potentially render the cancer cells sensitive to radiotherapy $(17,21)$. Mechanistically, isoflavones inhibit the activation of tyrosine kinase growth factor receptors and matrix metalloproteinases (MMPs) that are upregulated in glioblastoma and drive uncontrolled proliferation of tumors $(10,11,22-26)$.

Based on several studies on the anticancer potential of isoflavones, we hypothesize that biochanin A as a DNA repair inhibitor augments the cytotoxic activity of temozolomide, inhibiting cell proliferation and modulating cell metabolism in human glioblastoma cells, U-87 MG and T98 G.

\section{Materials and Methods}

Cell culture and drug treatments. Human glioblastoma cell lines, U87 MG and T98 G were obtained from American Type Culture Collection (Manassas, VA, USA) and were cultured as previously described (11).

Stock solutions of temozolomide and biochanin A (SigmaAldrich, St. Louis, MO, USA) were prepared in DMSO (SigmaAldrich) at $100 \mathrm{mM}$ each. Fresh dilutions were prepared from stock solutions prior to the experiments. The final concentration of DMSO in the culture medium did not exceed $0.07 \%(\mathrm{v} / \mathrm{v})$.

Cell viability and cell count assay. U-87 MG cells were plated in a 96-well plate with 2500 cells/well and treated with different concentrations of biochanin A and temozolomide. The assay was performed as previously described (11).

For cell count assay, U-87 MG cells $\left(10 \times 10^{3}\right.$ per well $)$ were plated in 24-well plates and treated with biochanin $\mathrm{A}$ and temozolomide alone or in combination for $72 \mathrm{~h}$. After treatment, the cells were trypsinized and counted on a Beckman $\mathrm{Z}$ series coulter counter (Beckman Coulter, Inc., Brea, CA, USA).

Colony formation assay. U-87 MG cells $\left(1 \times 10^{3}\right.$ cells per well) were cultured and treated with $70 \mu \mathrm{M}$ of biochanin A (B70) and temozolomide (T70), alone or in combination and incubated at $37^{\circ} \mathrm{C}$ in a Heracell $150 \mathrm{i} \mathrm{CO}_{2}$ incubator (Thermo Fisher Scientific, Waltham, MA, USA) with $5 \% \mathrm{CO}_{2}$ (v/v) overnight. The cells were maintained under similar growth conditions with medium replenished at regular intervals until $\sim 80 \%$ confluency was achieved. The cells were stained with crystal violet (in methanol) for $10 \mathrm{~min}$ and washed with distilled water. The plates were dried at room temperature and pictures were taken.

Hoechst staining assay. Both U-87 MG and T98 G cells were seeded $\left(10 \times 10^{3}\right)$ in a 24 -well plate and incubated overnight at $37^{\circ} \mathrm{C}$ in a $5 \% \mathrm{CO}_{2}(\mathrm{v} / \mathrm{v})$ incubator. The cells were treated with biochanin A $(70 \mu \mathrm{M})$ and/or temozolomide $(70 \mu \mathrm{M})$ for $72 \mathrm{~h}$. After treatment, the medium was aspirated and cells stained with a solution of Hoechst 33342 (Molecular Probes, OR, USA). The cells were incubated in dark for 15-30 min before fluorescent images were captured using Evos FL cell imaging system (Life technologies, Carlsbad, CA, USA) using 20× magnification.

Cell cycle analysis. About $2 \times 10^{4}$ cells per well were seeded in a 12-well plate and treated with $70 \mu \mathrm{M}$ biochanin A and temozolomide and incubated for $48 \mathrm{~h}$. The cells were harvested and fixed with ice cold $70 \%$ ethanol for $\sim 1 \mathrm{~h}$ at $4^{\circ} \mathrm{C}$. The fixed cells were washed twice with PBS, treated with $50 \mu \mathrm{l}$ of $100 \mu \mathrm{g} / \mathrm{ml}$ Ribonuclease A and $30 \mu \mathrm{l}$ of PI (Stock of $50 \mu \mathrm{g} / \mathrm{ml}$ ). The cells were kept at room temperature in dark for $30 \mathrm{~min}$ and analyzed for quantitation of DNA content and assessment of cell cycle distribution using the flow cytometer Guava easyCyte (Millipore Sigma, Burlington MA, USA).

\section{Cell metabolism studies}

Mito Stress Test. U-87 MG and T98 G cells were treated with biochanin $\mathrm{A}(70 \mu \mathrm{M})$ and temozolomide $(70 \mu \mathrm{M})$ individually or in combination, and incubated for $72 \mathrm{~h}$. After incubation, $3 \times 10^{4}$ cells per well were plated in an 8-well XFp cell culture miniplate (Seahorse Bioscience, Agilent, Santa Clara, CA, USA) and incubated overnight at $37^{\circ} \mathrm{C}$ in a $5 \% \mathrm{CO}_{2}(\mathrm{v} / \mathrm{v})$ incubator. After incubation, the growth medium in the miniplate was replaced with the assay medium (XF basal medium containing $1 \mathrm{mM}$ sodium pyruvate, $10 \mathrm{mM}$ D-Glucose \& $2 \mathrm{mM}$ L-Glutamine, $\mathrm{pH}$ 7.4), and incubated in a non- $\mathrm{CO}_{2}$ incubator at $37^{\circ} \mathrm{C}$ for $\sim 1 \mathrm{~h}$. The stock compounds rotenone/antimycin A, carbonyl cyanide-4 (trifluoromethoxy) phenylhydrazone (FCCP) and oligomycin were prepared by appropriately diluting the compounds with the assay medium. The stock compounds were loaded into the cartridge (Seahorse Bioscience, Agilent) hydrated with the XF calibrant to achieve final concentrations of $1.0 \mu \mathrm{M}$ oligomycin, $0.5 \mu \mathrm{M}$ FCCP and $0.5 \mu \mathrm{M}$ rotenone/antimycin $\mathrm{A}$. The oxygen consumption rate (OCR) and extracellular acidification rates (ECAR) were monitored using Seahorse XFp Extracellular Flux Analyzer (Seahorse Bioscience, Agilent).

XF-PMP (Plasma Membrane Permeabilizer) assay. U-87 MG and T98 G cells were treated with biochanin $\mathrm{A}(70 \mu \mathrm{M})$ and temozolomide $(70 \mu \mathrm{M})$ alone or in combination, and incubated for $72 \mathrm{~h}$. The cells were plated at a density of $3 \times 10^{4}$ cells per well in an 8 -well XFp cell culture miniplate (Seahorse Bioscience, Agilent) and incubated overnight at $37^{\circ} \mathrm{C}$ in a $5 \% \mathrm{CO}_{2}(\mathrm{v} / \mathrm{v})$ incubator. To evaluate the integrity of Complexes I-IV of the mitochondrial respiratory chain of the cells, OCR was measured as recommended by the manufacturer after cell permeabilization with $1 \mathrm{nM}$ PMP reagent (Seahorse Bioscience, Agilent). Briefly, $10 \mathrm{mM}$ pyruvate and $1 \mathrm{mM}$ malate were used as substrates, in addition to $4 \mathrm{mM}$ ADP. The final concentrations of the compounds injected in the cartridge were: port $\mathrm{A}, 2 \mu \mathrm{M}$ rotenone; port $\mathrm{B}, 10 \mathrm{mM}$ succinate; port $\mathrm{C}, 2 \mu \mathrm{M}$ antimycin $\mathrm{A}$; port $\mathrm{D}, 150 \mathrm{mM}$ ascorbate plus $2 \mathrm{mM}$ TMPD. The protocol and algorithm program for XF-PMP assay were designed using wave 2.4 software. The assay was repeated at least 2 times for each cohort.

Western Blot analysis. Western blot analysis was performed as described earlier $(10,11)$ to evaluate the effect of temozolomide and biochanin A (alone and in combination) on the expression of cell signaling proteins like ERK, p-ERK, AKT, p-AKT, EGFR, c-myc. Equal amounts of protein $(15 \mu \mathrm{g})$ were loaded into the wells in a sodium dodecyl sulfate poly acrylamide gel (SDS-PAGE) and the 


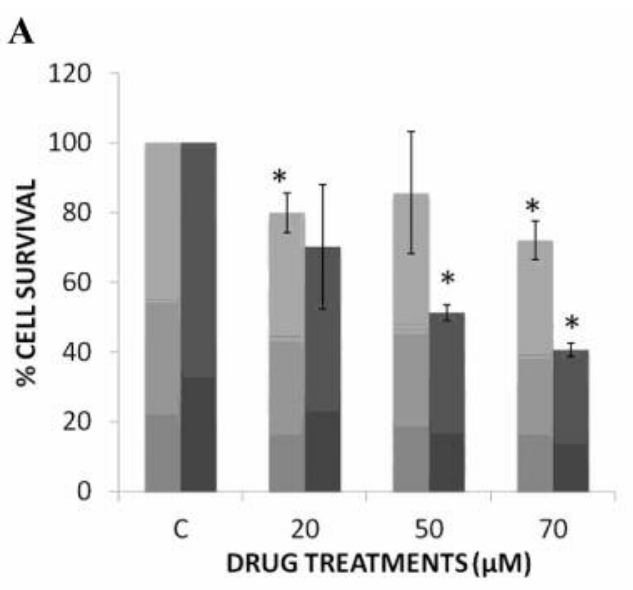

= TEMOZOLOMIDE

- BIOCHANINA
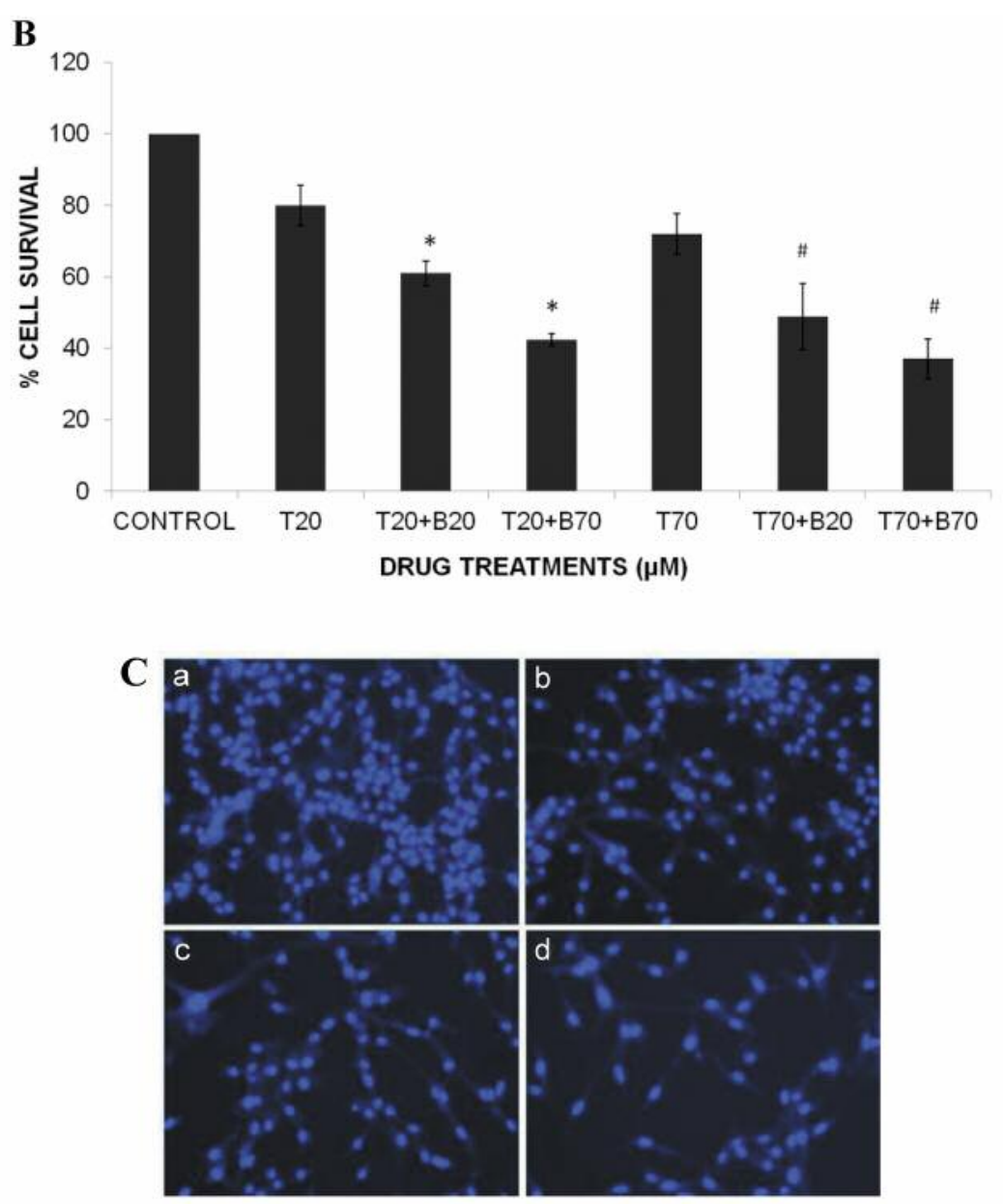

Figure 1. Cell viability studies. A. Cell count assay of U-87 MG cells treated with temozolomide and biochanin A for 72 h. $n=3$, * $\leq \leq 0.05$ versus control. B. Cell count assay of U-87 MG cells treated with temozolomide, biochanin A alone and in combination. Temozolomide combined with biochanin A was more effective in decreasing cell count of $U-87 \mathrm{MG}$ cells. $n=3, * p \leq 0.05$ versus control, @ $p \leq 0.05$ versus T20, \#p $\leq 0.05$ versus T70. C. Fluorescent images of U-87 MG cells treated with temozolomide and biochanin A (a: Control, b: Biochanin A $70 \mu M, c:$ Temozolomide $70 \mu M$, d: Biochanin A $70 \mu M+$ Temozolomide $70 \mu M$ ) for $72 \mathrm{~h}$ in a Hoechst assay. Combination of temozolomide and biochanin A (D) significantly reduced the number of live cells.

proteins were separated by electrophoresis. The relative expression of these proteins was normalized with $\beta$-actin. All antibodies were obtained from Santa Cruz Biotechnology (Santa Cruz, CA, USA).

Gelatin zymography. The gelatinolytic activity of MMPs (MMP-2 and MMP-9) was analyzed in U-87 MG cells treated with different drug concentrations and incubated at $37^{\circ} \mathrm{C}$ for $72 \mathrm{~h}$. The serum-free conditioned medium was collected, centrifuged and the resultant supernatant was freeze-dried and reconstituted with distilled water before loading it $(30 \mu \mathrm{l})$ into commercially available zymogram gels (BioRad, Hercules, CA, USA). The samples were further processed as previously described (10).

Statistical analysis. The data were analyzed by one-way ANOVA (using KaleidaGraph version 4.03), followed by Dunnett's post-hoc test. $p$-Value of $<0.05$ was considered statistically significant in each analysis.

\section{Results}

Temozolomide and biochanin A reduced GBM cell viability. Temozolomide decreased the viability of U-87 MG cells in a dose-dependent manner. The cell count assay of U-87 MG cells clearly showed that both temozolomide and biochanin A decreased the percentage count of $\mathrm{U}-87 \mathrm{MG}$ cells in a dose-responsive manner (Figure 1A). Moreover, biochanin A significantly potentiated the effect of temozolomide in combination, showing an optimal inhibition at $70 \mu \mathrm{M}$ (Figure 1B). These observations were further consolidated by the results of Hoechst assay and colony formation assay, clearly showing the efficacy of the combination treatment in inhibiting U-87 MG cells, compared to either of the drugs alone (Figure 1C). 

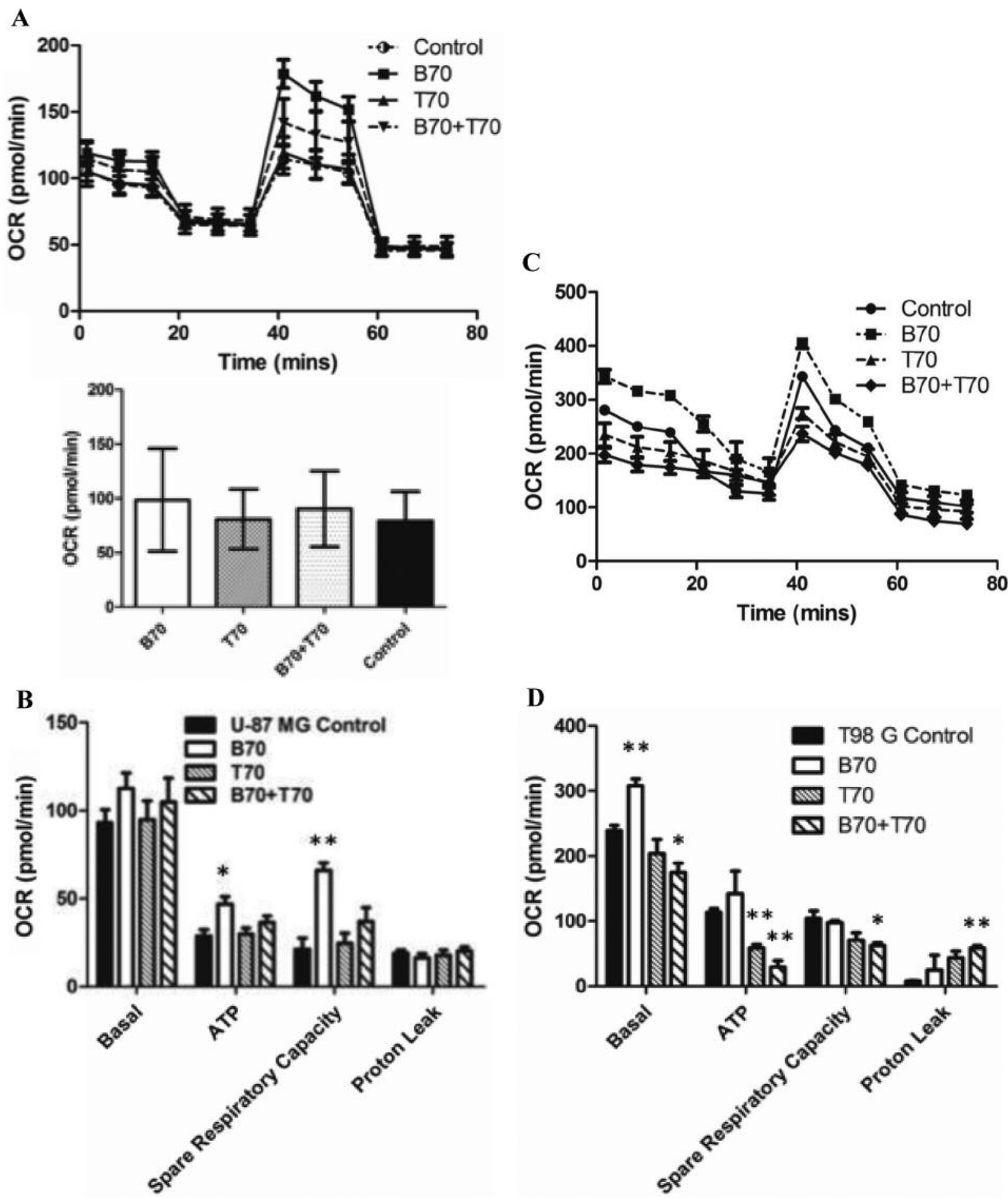

Figure 2. Cell metabolism studies (Mito stress test). A. The effect of treatment of biochanin A (B70), temozolomide (T70) and the combination treatment $(B 70+T 70)$, on the rate of mitochondrial respiration $(O C R)$ in $U-87 M G$ cells after $72 \mathrm{~h}$, as determined by the Mito stress test. The B70 treatment showed a maximal increase in OCR, but a clear increase in the OCR can be observed on combination treatment compared to temozolomide alone. $B$. The combination treatment did not show any effect on the process of ATP production, spare respiratory capacity or proton leak in the U-87 MG cells, while B70 treatment seems to increase the ATP production and spare respiratory capacity of the cells $\left({ }^{*} p<0.05\right.$ and $\left.* * p<0.01\right)$. C. Effect of combination treatment $(B 70+T 70)$, biochanin $A(B 70)$ and temozolomide (T70), on the rate of mitochondrial respiration $(O C R)$ in T98 G cells after $72 \mathrm{~h}$. A decrease in the OCR of cells I is seen on combination treatment compared to the B70 and T70 alone. D. The combination treatment clearly caused a decrease in ATP production and the spare respiratory capacity while increasing the proton leak in T98 $G$ cells $\left({ }^{*} p<0.05\right.$ and $\left.* * p<0.01\right)$. 

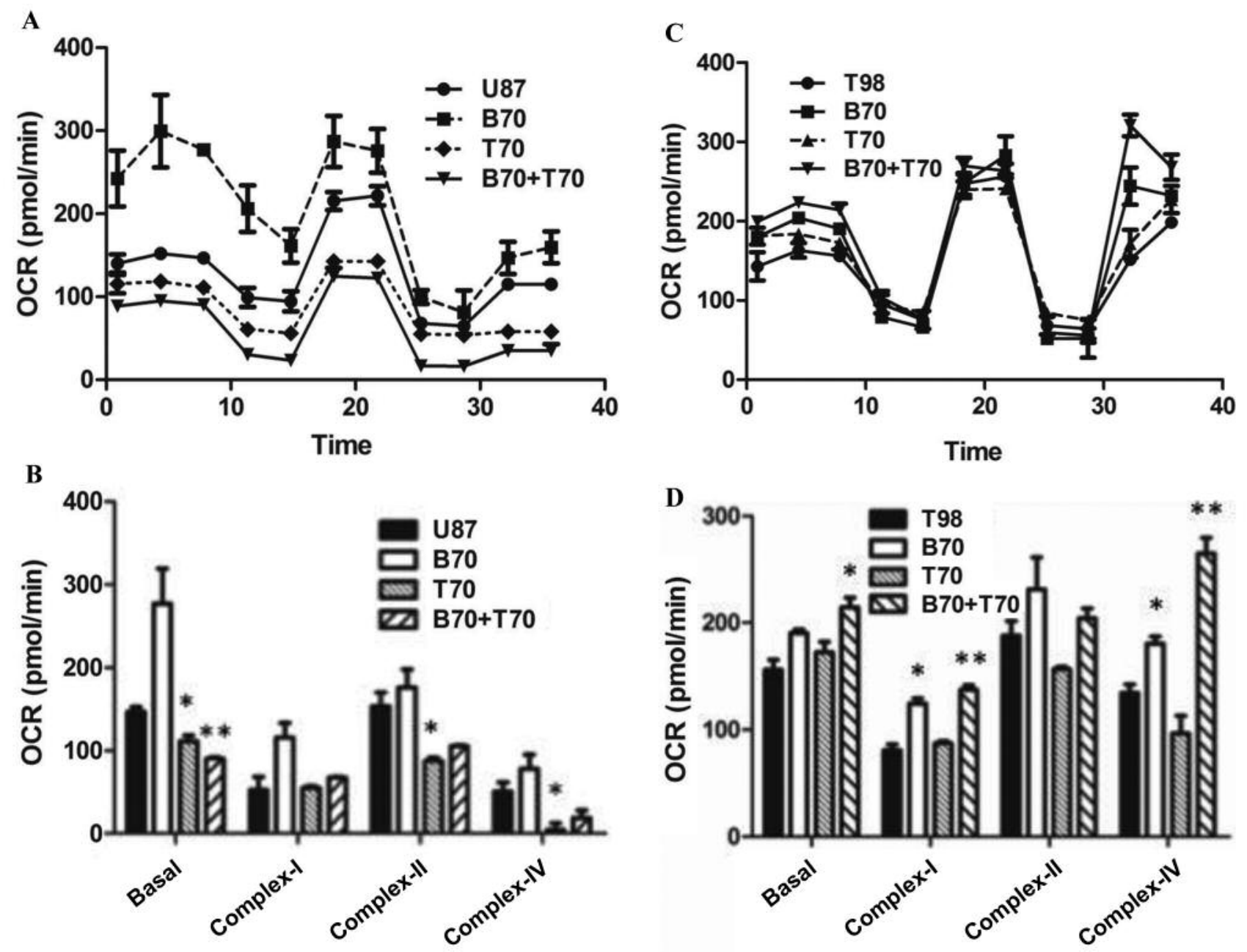

Figure 3. Cell metabolism studies (PMP assay). A. The XF-PMP assay was performed on U-87 MG cells treated with Biochanin A, Temozolomide, \& the combination of both the drugs. The cells were permeabilized with $1 \mathrm{nM} X F-P M P$ reagent and the respiratory chain complexes were probed with specific substrates, inhibitors and uncouplers to evaluate the effect of treatments on mitochondrial respiration. The combination treatment led to a decrease in the OCR compared to other treatments. B. A clear decrease in mitochondrial respiration is observed and is mediated mainly through complex-IV inhibition in temozolomide and the combination treatment $\left({ }^{*} p<0.05\right.$ and $\left.{ }^{* *} p<0.01\right)$. All the treatments were compared to the control. $C$. The T98 $G$ cells were treated with Biochanin A, Temozolomide, \& the combination treatment and XF-PMP assay was performed. The cells were permeabilized with 1 nM XF-PMP reagent. The effect on mitochondrial respiration was evaluated by probing the respiratory chain complexes with specific substrates, inhibitors and uncouplers. D. The combination treatment led to an increase in the activity of all the complexes (especially complex I and IV) while the other treatments had no significant effect on the respiratory complex-mediated mitochondrial respiration in T98 G cells $\left({ }^{*} p<0.05\right.$ and $\left.* * p<0.01\right)$.

The combination treatment however, did not show any significant inhibitory effect on the T98 G cell count.

Combination of biochanin A and temozolomide increased oxidative phosphorylation in U-87 MG cells. Following the 72-h drug treatment of U-87 MG cells, the changes in the metabolic phenotype of cells were evaluated by monitoring the OCR and ECAR in real time. The basal mitochondrial respiration of the cells in combination treatment showed no significant difference relative to control. To evaluate the maximal mitochondrial respiration of cells, FCCP, a mitochondrial oxidative phosphorylation uncoupler was used. The combination treatment showed an increase in maximal mitochondrial respiration to $\sim 142 \mathrm{pmol} / \mathrm{min}$ compared to $\sim 119 \mathrm{pmol} / \mathrm{min}$ in $\mathrm{T} 70$ alone and $\sim 114$ $\mathrm{pmol} / \mathrm{min}$ in control cells (Figure 2A). Cells treated with B70 alone had a maximal respiration of $\sim 178 \mathrm{pmol} / \mathrm{min}$. The combination treatment or T70 alone did not induce a 

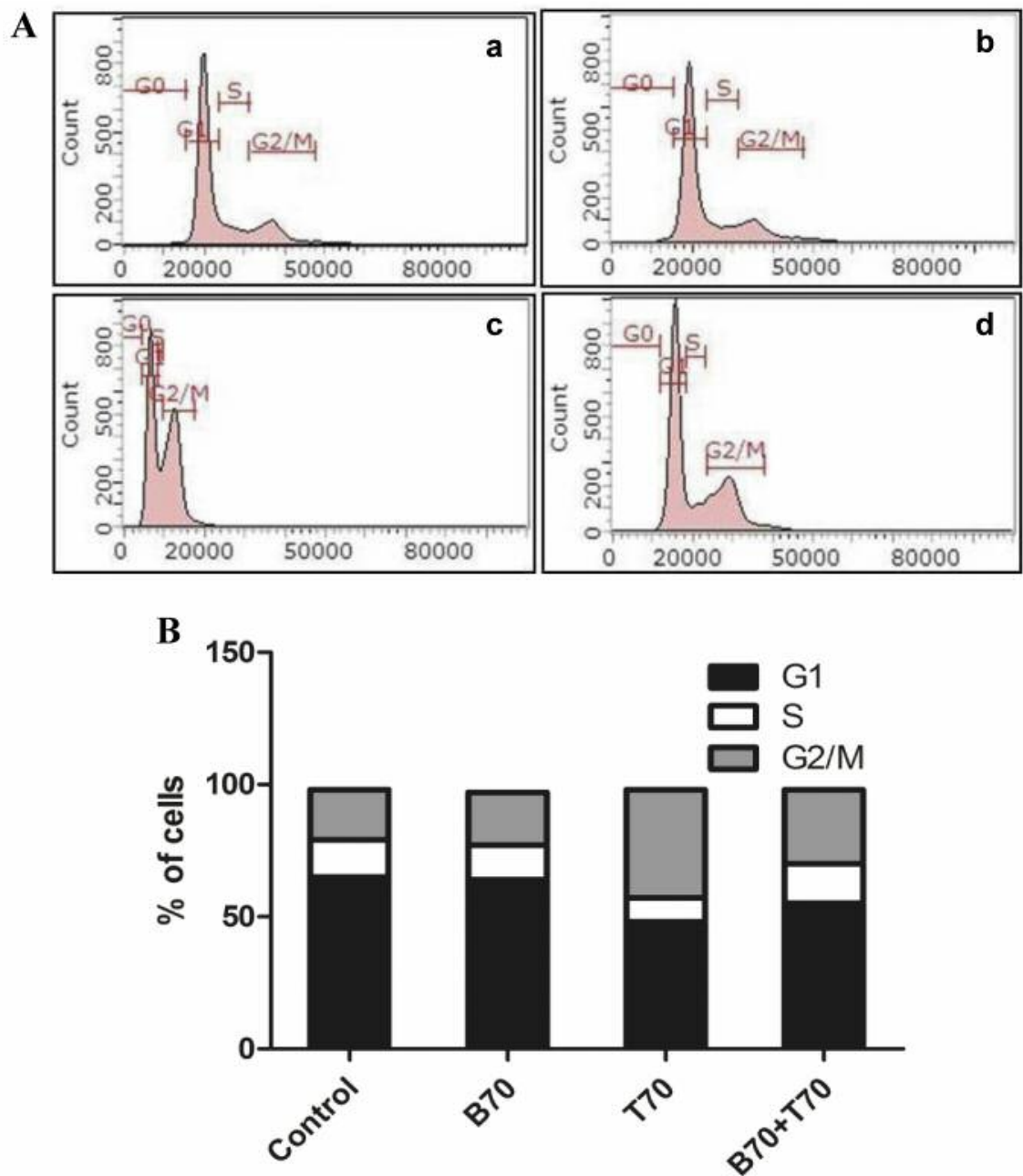

Figure 4. Cell-cycle analysis. A. Cell-cycle analysis of U-87 MG cells treated with: $a$ : Control, $b$ : Biochanin A $70 \mu M, c:$ Temozolomide $70 \mu M, d$ : Biochanin A $70 \mu M+$ temozolomide $70 \mu M$, for $48 \mathrm{~h}$. A clear increase in the $G_{1}$ phase arrest is observed on combination treatment of cells. $B$. Cell cycle distribution of U-87 MG cells treated with biochanin A and temozolomide alone and in combination for $48 \mathrm{~h}$.

significant effect on the respiratory capacity and proton leak in the cells (Figure 2B). However, cells treated with the combination of compounds showed a significantly decreased glycolysis (ECAR) compared to control cells.

Oxidative phosphorylation decreased in T98 G cells after combination treatment. In T98 G cells, the combination and T70 alone treatments showed a decrease in maximal respiration to $\sim 236 \mathrm{pmol} / \mathrm{min}$ and $\sim 274 \mathrm{pmol} / \mathrm{min}$ respectively, from $\sim 343 \mathrm{pmol} / \mathrm{min}$ in control cells (Figure $2 \mathrm{C})$. The cells treated with B70 showed a maximal respiration of $\sim 405 \mathrm{pmol} / \mathrm{min}$. However, the combination treatment was observed to cause a decrease in basal OCR, ATP production and spare respiratory capacity while increasing the proton leak in the cells (Figure 2D). Furthermore, the variation in the ECAR of control cells and treated cells was very insignificant and the treated cells showed a decreased ECAR compared to control cells.

Combination treatment altered mitochondrial respiration by inhibiting Complex IV activity in permeabilized U-87 MG cells but not in T98 G cells. The U-87 MG cells were permeabilized with $1 \mathrm{nM}$ PMP reagent and evaluated for changes in OCR, after treatment with biochanin A and temozolomide alone and in combination. A clear decrease in OCR was observed on combination treatment and with T70 alone compared to the control (Figure 3A). This decrease in mitochondrial respiration was primarily mediated through respiratory complex IV activity (Figure 3B).

However, in the permeabilized T98 G cells, a mild increase in the OCR was observed after combination treatment (Figure 3C). No significant decrease was observed in mitochondrial 
respiration and the combination treatment did not show any inhibitory effect on any of the respiratory chain complexes. Conversely, the combination treatment seemed to increase the activities of complex I and IV (Figure 3D).

The combination treatment repealed $G_{2} / M$ phase arrest. In U-87 MG cells, temozolomide exerted a significant induction of $\mathrm{G}_{2} / \mathrm{M}$ phase arrest after $48 \mathrm{~h}$, while no alteration in cell cycle distribution was observed with biochanin A. However, on combination treatment, biochanin A seemed to rescind the $\mathrm{G}_{2} / \mathrm{M}$ phase arrest, showing an increase in $\mathrm{G}_{1}$ phase arrest in the cells (Figure 4A and B). The T98 G cells, also showed a mild increase in $\mathrm{G} 1$ phase arrest, after $48 \mathrm{~h}$ of combination treatment.

Combination treatment decreased the expression of cell signaling proteins. Western blot analysis was performed to determine the effect of temozolomide and biochanin $\mathrm{A}$ on the expression of cell signaling proteins. The results demonstrated that compared to either biochanin A or temozolomide alone, the combination treatment of biochanin A and temozolomide decreased the levels of p-ERK, p-AKT, EGFR, and c-myc and up-regulated the expression of phosphorylated-p53 (p-p53) (Figure 5A and B). Temozolomide alone was not effective in inhibiting the protein expression of EGFR or c-myc and the phosphorylation of either ERK or AKT (Figure 5A).

Biochanin A enhanced the inhibitory effect of temozolomide on MMPs. Western blot analysis to determine the expression of MMPs in U-87 MG cells showed that the combination treatment of biochanin A and temozolomide decreased the protein expression of MT1-MMP (Figure 5B) and inhibited the activity of MMP-2 (Figure 5C) compared to either biochanin A or temozolomide alone.

\section{Discussion}

GBM is one of the most difficult brain cancers to treat. The survival rate of patients with GBM remains low despite a multimodal approach to treatment (27-30). The efficacy of alkylating drugs like temozolomide depends largely on the DNA repair pathways, pro-survival pathways like PI3K/AKT and pro-invasive enzymes that are upregulated in GBM (11, 31-35). Isoflavones can inhibit tumor progression by impeding DNA repair and cell signaling pathways like Wnt and Nf-kB by inhibiting topoisomerase II and by acting as antioxidants (10, 36-39). Hence, combining temozolomide with biochanin A, could be an effective way to improve the efficacy of temozolomide in GBM treatment (10, 38-40).

A clear decrease in viability of U-87 MG cells was observed with both temozolomide and biochanin A. However, the effect of biochanin A could be attributed to the inherent nature of isoflavones, to increase the reducing
A
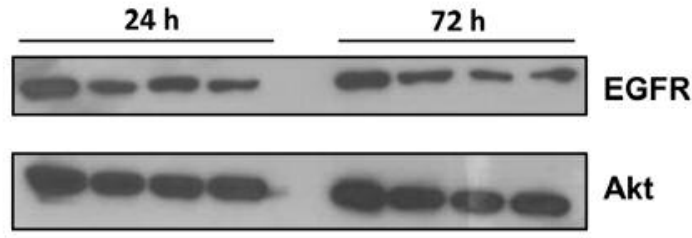

Akt

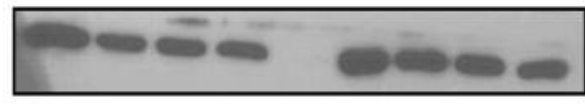

p-Akt

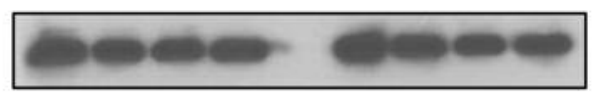

ERK

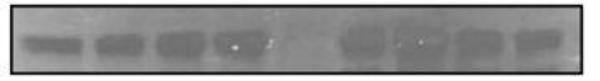

p-ERK
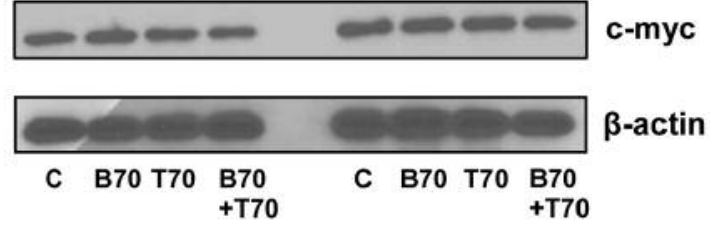

B

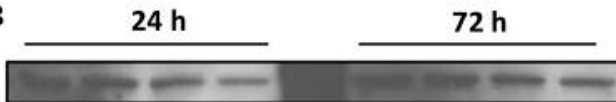

MT-MMP1

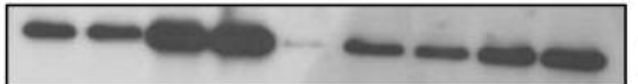

p-p53

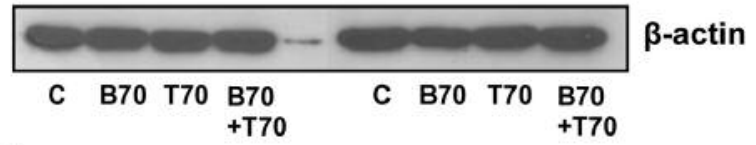

C

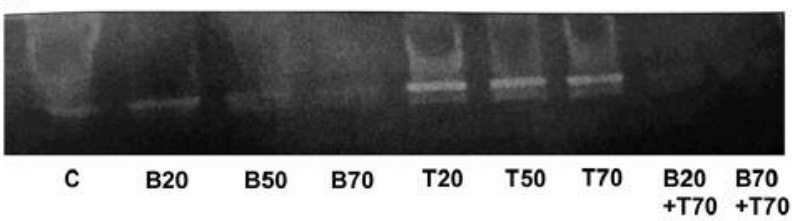

Figure 5. Cell signaling and invasion studies. A. Western blot analysis of cell signaling proteins: Human glioblastoma $U-87$ MG cells were treated with biochanin A $70 \mu \mathrm{M}$, temozolomide $70 \mu \mathrm{M}$ alone and in combination for $24 h$ and $72 h$. Cell lysates were prepared and western blot analysis was carried out. Temozolomide at $70 \mu \mathrm{M}$ in combination with biochanin $A$ at $70 \mu M$, significantly inhibited protein expression of EGFR, $p$-ERK, $p$-AKT and c-myc. $\beta$-actin was used as a loading control. B. Western blot analysis of MT-MMP1 and p-p53: Biochanin A at $70 \mu M$ in combination with $70 \mu M$ temozolomide significantly inhibited the expression of MT-MMP1, while p53 expression is increased compared to temozolomide alone in human glioblastoma $(U-87 \mathrm{MG})$ cells treated for $72 \mathrm{~h}$. The protein expression was normalized with $\beta$-actin. C. Gelatin zymography analysis of the activity of MMP-2: Temozolomide at all concentrations did not decrease the activity of MMP-2 in glioblastoma cells treated for $72 \mathrm{~h}$ in serum-free media. Combination treatment with $70 \mu \mathrm{M}$ biochanin $\mathrm{A}$ and $70 \mu \mathrm{M}$ temozolomide led to a significant inhibition in the activity of MMP-2 as seen here with a substantial reduction in the intensity of white bands. 
capacity of mitochondrial enzymes which intensifies the formation of formazan crystals in MTT $(23,27)$. Thus, with cell count assay that is devoid of interference from any dye or colored chemicals, an optimal inhibition was observed on combination treatment of biochanin $\mathrm{A}, 70 \mu \mathrm{M}$ and temozolomide, $70 \mu \mathrm{M}$ in U-87 MG cells. The combination treatment however, showed little effect on T98G cells, which are known to be intrinsically resistant to temozolomide (7). The viability results were further confirmed by the Hoechst assay, that showed a significant change in nuclear morphology where only a decrease in the number of live cells was recorded (28).

GBM cells reportedly rely on the glycolytic pathway for ATP generation even in the presence of oxygen, a metabolic state termed as "aerobic glycolysis" $(3,29)$, which leads to an increased rate of glucose consumption relative to normal cells $(29,30,41)$. We investigated the metabolic state of GBM cells by measuring the rate of glycolysis (ECAR) and mitochondrial respiration (OCR) in real time. In both the cells, the biochanin treatment showed an increase in OCR compared to the other treatments. Isoflavones such as biochanin A, reportedly promote mitochondrial biogenesis i.e. the production of functional mitochondria, by increasing PGC- $1 \alpha$ protein expression $(42,43)$. Hence, the increase in intracellular ATP observed in both the cells on treatment with biochanin A indicates, that the mitochondrial biogenesis and respiration is coupled to ATP synthesis $(42,43)$. In U-87 MG cells, the combination treatment showed a clear shift in metabolic phenotype from a glycolytic to an oxidative phosphorylation compared to the control and temozolomide alone. Furthermore, a clear inhibition of glycolysis is observed in U-87 MG cells, driving cells to use mitochondria to make up for the ATP deficit. The sustained levels of OCR after the FCCP injection implies that there is likely to be a pool of other oxidizable substrates for respiration. Recent studies have suggested the importance of restoring normal oxidative phosphorylation in cancer cells, to not only inhibit cell proliferation, but also impede the metastatic potential of malignant cells $(28-30,42)$.

The combination treatment did not show any effect on the metabolic phenotype of $\mathrm{T} 98 \mathrm{G}$ cells and the treatment weakly inhibited glycolysis. The sudden drop in maximal respiration after FCCP injection further suggests oxidation of all the available substrates for respiration. The decrease in spare capacity may result from the inhibition of a substrate that could have been oxidized otherwise $(29,30,42)$. The combination treatment in U-87 MG cells seems to make the mitochondrial metabolism more bioenergetic, leading to decreased metabolic intermediates and inhibition of cell proliferation $(29,42)$. The glycolytic metabolism of cancer cells is reported to strongly correlate with resistance to radiotherapy as the ionizing radiations cause the uncoupling of oxidative phosphorylation leading to increased glycolysis
$(32,38,42)$. Hence, the reversal of glycolytic phenotype in our study demonstrates that the combination treatment can possibly augment the efficacy of radiation therapy by inducing oxidative stress and lowering the acid production $(29,32,42)$.

Another problem in GBM treatment, is the chemoresistance developed by a subset of cancer cells called cancer stem cells (CSCs), that are mostly driven by mitochondrial respiration $(42,44)$. Hence, inhibiting the mitochondrial function in CSCs could potentially lead to elimination of chemoresistance and recurrence of the disease. We evaluated the mitochondrial function of GBM cells permeabilized with $1 \mathrm{nM}$ PMP reagent, by probing the activity of respiratory complexes by the XFPMP assay. Again, the increase in OCR of permeabilized U87 MG and T98 G cells, on treatment with B70, could be attributed to the antioxidative properties of biochanin $\mathrm{A}$. The decrease in OCR of U-87 MG cells after treatment with temozolomide alone and in combination, is primarily mediated through the inhibition of respiratory complex IV activity. Complex IV is the terminal component of the electron transport chain (ETC) that controls the efficient coupling of mitochondria and thus ROS production by regulating the electron flux capacity of ETC (42-44). In GBM patients, an increased activity of cytochrome $\mathrm{C}$ oxidase (complex IV) has been associated with the development of resistance to temozolomide and a shorter median survival time $(36,42,44)$. Although the exact mechanism of temozolomide mediated decrease in the complex IV activity remains to be elucidated in U87 MG cells, the increase in the complex IV activity in temozolomide resistant $\mathrm{T} 98 \mathrm{G}$ cells indicates that the complex IV has a pivotal role in the acquisition of chemoresistance to temozolomide.

Both genistein and biochanin A, have shown anticancer and anti-invasive potential by inhibiting matrix-degrading enzymes MMP-2, MMP-9 and UPA/uPAR in GBM $(10,37)$. Overexpression of EGFR and EGFRvIII in glioblastoma cells is associated with their increased proliferation and invasion $(31,35)$. We observed that biochanin A augments the effect of temozolomide in inhibiting EGFR and other active forms of cell proliferation and survival proteins, ERK and AKT, i.e. pERK and p-AKT while increasing the expression of tumor suppressor, p-p53. We also observed an increase in the expression of pAKT in U-87 MG cells in high glucose media, as compared to that in the low glucose media. Temozolomide treated cells are known to up-regulate p53-dependent pro-apoptotic proteins, Noxa and Bax (34). Thus, the increased expression of active p53 on combination treatment, might be responsible for improving the cytotoxic effects of temozolomide on cell cycle arrest or senescence $(33,40)$. Our cell-cycle studies also showed that, biochanin A improves the potency of temozolomide mainly by impeding the temozolomide-induced $\mathrm{G}_{2} / \mathrm{M}$ phase arrest in the cells. MMPs (MMP-2 and MMP-9), that play an important role in GBM 
invasion, are overexpressed in malignant glioblastoma and are important prognostic markers in pre-treatment and posttreatment phases of GBM $(10,37)$. Previously, we have also shown that, biochanin A effectively inhibited the expression of HIF- $1 \alpha$ and VEGF proteins, key players in angiogenesis $(10,11)$. Here, we clearly observed that biochanin A enhances the inhibitory potential of temozolomide and decreases protein expression of MMP-2 (72 KD) and its activator MT-MMP1. Hence, our studies indicate that, biochanin A might have a dual role in inhibiting both angiogenesis and invasion in GBM. Many reports on combination therapies of MMPs inhibitor and temozolomide highlight the importance of targeting MMPs (38).

In conclusion, this study summarizes the effects of combination treatment of temozolomide and biochanin A on GBM progression. Although, biochanin A showed an increase in the levels of OCR compared to other treatments, overall the combination treatment exhibited an enhanced anticancer effect on GBM cells. Here, biochanin A considerably potentiated the cytotoxicity of temozolomide and enhanced inhibition of cell signaling and invasion pathways, while also restoring the metabolic phenotype of cells to mitochondrial respiration. The results provide a rationale for evaluating combination therapies with biochanin A in vivo and support the design of new and better therapies for the treatment of an aggressive, fatal tumors like GBM.

\section{Acknowledgements}

This work was supported by Jefferson College of Pharmacy (JCP), and The Mountain States Tumor and Medical Research Institute (MSTMRI). The Authors would like to thank Jennifer Wilson, Medical Writer/Editor, Center for Teaching and Learning, Thomas Jefferson University for editing the manuscript.

\section{References}

1 Ostrom QT, Gittleman H, Liao P, Rouse C, Chen Y, Dowling J, Wolinsky Y, Kruchko C and Barnholtz-Sloan J: CBTRUS Statistical Report: Primary brain and central nervous system tumors diagnosed in the United States in 2007-2011. Neuro Oncol 16: 41-463, 2014.

2 Wrensch M, Minn Y, Chew T, Bondy M and Beregr MS: Epidemiology of primary brain tumors: Current concepts and review of the literature. Neuro Oncol 4: 278-299, 2002.

3 Kesari S: Understanding glioblastoma tumor biology: the potential to improve current diagnosis and treatments. Semin Oncol 38: S2-10, 2011.

4 Villano JL, Seery TE and Bressler LR: Temozolomide in malignant gliomas: current use and future targets. Cancer Chemother Pharmacol 64: 647-655, 2009.

5 Knizhnik AV, Roos WP, Nikolova T, Quiros S, Tomaszowski KH, Christmann M and Kaina B: Survival and death strategies in glioma cells: autophagy, senescence and apoptosis triggered by a single type of temozolomide-induced DNA damage. PloS One 8: 1-12, 2013.
6 Zhang J, Stevens MFG and Bradshaw TD: Temozolomide: mechanisms of action, repair and resistance. Curr Mol Pharmacol 5: 102-114, 2012.

7 Lee SY: Temozolomide resistance in glioblastoma multiforme. Genes Dis 3: 198-210, 2016.

8 Hillman GG and Singh-Gupta V: Soy isoflavones sensitize cancer cells to radiotherapy. Free Radic Biol Med 51: 289-298, 2011.

9 Yoshimoto K, Mizoguchi M, Hata N, Murata H, Hatae R, Amano T, Nakamizo A and Sasaki T: Complex DNA repair pathways as possible therapeutic targets to overcome temozolomide resistance in glioblastoma. Front Oncol 2: 1-8, 2012.

10 Puli S, Lai CKJ and Bhushan A: Inhibition of matrix degrading enzymes and invasion in human glioblastoma (U87MG) cells by isoflavones. J Neurooncol 79: 135-142, 2006.

11 Puli S, Jain A, Lai CKJ and Bhushan A: Effect of Combination Treatment of Rapamycin and Isoflavones on mTOR Pathway in Human Glioblastoma (U87) Cells. Neurochem Res 35: 986-993, 2010.

12 Prasad G, Sottero T, Yang X, Mueller S, James CD, Weiss WA, Polley MY, Ozawa T, Berger MS, Aftab DT, Prados MD and Haas-Kogan DA: Inhibition of $\mathrm{PI} 3 \mathrm{~K} / \mathrm{mTOR}$ pathways in glioblastoma and implications for combination therapy with temozolomide. Neuro Oncol 13(4): 384-392, 2011.

13 Sehdev V, Lai CKJ and Bhushan A: Biochanin A Modulates Cell Viability, Invasion, and Growth Promoting Signaling Pathways in HER-2-Positive Breast Cancer Cells. J Oncol 2009: 1-10, 2009.

14 Raffoul JJ, Heydari AR and Hillman GG: DNA Repair and Cancer Therapy: Targeting APE1/Ref-1 Using Dietary Agents. J Oncol 2012: 11, 2012.

15 Rice L, Samedi VG, Medrano TA, Sweeney CA, Baker HV, Stenstrom A, Furman J and Shiverick KT: Mechanisms of the growth inhibitory effects of the isoflavonoid biochanin A on LNCap cells and xenografts. Prostate 52: 201-212, 2002.

16 Block G, Patterson B and Subar A: Fruit, vegetables, and cancer prevention: a review of the epidemiological evidence. Nutr Cancer 18: 1-29, 1992.

17 Persky V and Van Horn L: Epidemiology of soy and cancer: perspectives and directions. J Nutr 125: 709S-712S, 1995.

18 Tedeschi-Blok N, Lee M, Sison JD, Miike R and Wrensch M: Inverse association of antioxidant and phytoestrogen nutrient intake with adult glioma in the San Francisco Bay Area: a casecontrol study. BMC Cancer 6: 148, 2006.

19 Akiyama T, Ishida J, Nakagawa S, Ogawara H, Watanabe S, Itoh N, Shibuya M and Fukami Y: Genistein, a specific inhibitor of tyrosine-specific protein kinases. J Biol Chem 262: 5592-5595, 1987.

20 Moon YJ, Sagawa K, Frederick K, Zhang S and Morris ME: Pharmacokinetics and bioavailability of the isoflavone biochanin A in rats. The AAPS journal 8: E433-442, 2006.

21 Wachsberger PR, Lawrence RY, Liu Y, Xia X, Andersen B and Dicker AP: Cediranib enhances control of wild type EGFR and EGFRvIII-expressing gliomas through potentiating temozolomide, but not through radiosensitization: implications for the clinic. J Neurooncol 105: 181-190, 2011.

22 Mimeault $\mathrm{M}$ and Batra SK: Complex oncogenic signaling networks regulate brain tumor-initiating cells and their progenies: pivotal roles of wild-type EGFR, EGFRvIII mutant and hedgehog cascades and novel multitargeted therapies. Brain Pathology 21: 479-500, 2011. 
23 Mayo LD, Dixon JE, Durden DL, Tonks NK and Donner DB: PTEN protects p53 from Mdm2 and sensitizes cancer cells to chemotherapy. J Biol Chem 277: 5484-5489, 2002.

24 Jiang Z, Pore N, Cerniglia GJ, Mick R, Georgescu MM, Bernhard EJ, Hahn SM, Gupta AK and Maity A: Phosphatase and tensin homologue deficiency in glioblastoma confers resistance to radiation and temozolomide that is reversed by the protease inhibitor nelfinavir. Cancer Res 67: 4467-4473, 2007.

25 Wang $\mathrm{Z}$ and Chen $\mathrm{H}$ : Genistein increases gene expression by demethylation of WNT5a promoter in colon cancer cell line SW1116. Anticancer Res 30: 4537-4545, 2010.

26 Sarkar FH, Li Y, Wang Z and Kong D: Cellular signaling perturbation by natural products. Cell Signal 21: 1541-1547, 2009.

27 Pagliacci MC, Spinozzi F, Migliorati G, Fumi G, Smacchia M, Grignani F, Riccardi C and Nicoletti I: Genistein inhibits tumour cell growth in vitro but enhances mitochondrial reduction of tetrazolium salts: a further pitfall in the use of the MTT assay for evaluating cell growth and survival. Eur J Cancer 29A: 15731577, 1993.

28 Swarnkar S, Singh S, Goswami P, Mathur R, Patro IK and Nath $\mathrm{C}$ : Astrocyte activation: a key step in rotenone induced cytotoxicity and DNA damage. Neurochem Res 37: 2178-2189, 2012.

29 Poteet E, Choudhury GR, Winters A, Li W, Ryou M, Liu R, Tang L, Ghorpade A, Wen Y, Yuan F, Keir ST, Yan H, Bigner DD, Simpkins JW and Yang SH: Reversing the Warburg Effect as a Treatment for Glioblastoma. J Biol Chem 288: 9153-9164, 2013.

$30 \mathrm{Ru}$ P, Williams TM, Chakravarti A and Guo D: Tumor metabolism of malignant gliomas. Cancers 5: 1469-1484, 2013.

31 Nicholas MK, Lukas RV, Jafri NF, Faoro L and Salgia R: Epidermal growth factor receptor - mediated signal transduction in the development and therapy of gliomas. Clin Cancer Res 12: 7261-7270, 2006.

32 Brown PD, Krishnan S, Sarkaria JN, Wu W, Jaeckle KA, Uhm JH, Geoffroy FJ, Arusell R, Kitange G, Jenkins RB, Kugler JW, Morton RF, Rowland KM Jr, Mischel P, Yong WH, Scheithauer BW, Schiff D, Giannini C and Buckner JC: Phase I/II trial of erlotinib and temozolomide with radiation therapy in the treatment of newly diagnosed glioblastoma multiforme: North Central Cancer Treatment Group Study N0177. J Clin Oncol 26: 5603-5609, 2008.

33 Hirose Y, Berger MS and Pieper RO: p53 effects both the duration of $\mathrm{G} 2 / \mathrm{M}$ arrest and the fate of temozolomide-treated human glioblastoma cells. Cancer Res 61: 1957-1963, 2001.
34 Zhang WB, Wang Z, Shu F, Jin YH, Liu HY, Wang QJ and Yang Y: Activation of AMP-activated protein kinase by temozolomide contributes to apoptosis in glioblastoma cells via p53 activation and mTORC1 inhibition. J Biol Chem 285: 40461-40471, 2010.

35 Zadeh G, Bhat KPL and Aldape K: EGFR and EGFRvIII in Glioblastoma: Partners in Crime. Cancer Cell 24: 403-404, 2013.

36 Friedmann-Morvinski D, Narasimamurthy R, Xia Y, Myskiw C, Soda Y and Verma IM: Targeting NF-kB in glioblastoma: A therapeutic approach. Sci Adv 2: e1501292, 2016.

37 Gabelloni P, Da Pozzo E, Bendinelli S, Costa B, Nuti E, Casalini F, Orlandini E, Da Settimo F, Rossello A and Martini C: Inhibition of metalloproteinases derived from tumours: new insights in the treatment of human glioblastoma. Neuroscience 168: 514-522, 2010

38 Trog D, Yeghiazaryan K, Fountoulakis M, Friedlein A, Moenkemann H, Haertel N, Schueller H, Breipohl W, Schild H, Leppert D and Golubnitschaja O: Pro-invasive gene regulating effect of irradiation and combined temozolomide-radiation treatment on surviving human malignant glioma cells. Eur $\mathbf{J}$ Pharmacol 542: 8-15, 2006.

39 Sarkar FH and Li YW: Targeting multiple signal pathways by chemopreventive agents for cancer prevention and therapy. Acta Pharmacol Sin 28: 1305-1315, 2007.

40 Filippi-Chiela EC, Thome MP, Bueno e Silva MM, Pelegrini AL, Ledur PF, Garicochea B, Zamin LL and Lenz G: Resveratrol abrogates the Temozolomide-induced G2 arrest leading to mitotic catastrophe and reinforces the Temozolomide-induced senescence in glioma cells. BMC Cancer 13: 147, 2013.

41 McNamara MG, Sahebjam S and Mason WP: Emerging biomarkers in glioblastoma. Cancers 5: 1103-1119, 2013.

42 Shen H, Hau E, Joshi S, Dilda PJ and McDonald KL: Sensitization of Glioblastoma Cells to Irradiation by Modulating the Glucose Metabolism. Mol Cancer Ther 14: 1794-1804, 2015.

43 Rasbach KA and Schnellmann RG: Isoflavones Promote Mitochondrial Biogenesis. J. Pharmacol Exp Ther 325: 536-543, 2008.

44 Oliva CR, Zhang W, Langford C, Suto MJ and Griguer CE: Repositioning chlorpromazine for treating chemoresistant glioma through the inhibition of cytochrome c oxidase bearing the COX4-1 regulatory subunit. Oncotarget 8: 37568-37583, 2017.

Received November 27, 2018

Revised December 3, 2018

Accepted December 6, 2018 